\title{
SYNTHESIS OF O-SUBSTITUTED BENZOPHENONES BY GRIGNARD REACTION OF 3-SUBSTITUTED ISOCOUMARINS
}

\author{
P. MANIVEL, S. MOHANA ROOPAN, F. NAWAZ KHAN*
}

* Chemistry Division, School of Science and Humanities,

VIT-University, Vellore 632 014, Tamil Nadu, India.

(Received: November 9, 2007 - Accepted: March 27, 2008)

\begin{abstract}
A series of o-substituted benzophenones was prepared by efficient method involving the 3-substituted isocoumarins and phenylmagnesium bromide. The reaction was completed in presence of anhydrous ether under dry ice-solvent bath in few hours with modest to good yields.
\end{abstract}

Key words: Isocoumarins, diketones, aryl, phenyl magnesium bromide, ether, dry ice.

\section{INTRODUCTION}

Synthetic and naturally occurring isocoumarins possess important biological properties. They are useful intermediates in the synthesis of a variety of carbocyclic and heterocyclic compounds ${ }^{1-6}$. In continuous of our interest in carbon-carbon bond formation reaction ${ }^{7-12}$, the present paper presents an efficient method to synthesize o-substituted benzophenones (2) from 3substitued isocoumarins (1) and phenyl magnesium bromide in presence of anhydrous ether under dry ice-solvent bath. Benzophenone and its derivatives are important intermediates in dyes, pharmaceutical and other chemical industries ${ }^{13-15}$. Benzophenone analogues synthesized by several scientists have been reported as effective anti-inflammatory agents ${ }^{16-18}$. Recently synthesis and structure-activity relationship of benzophenones as novel class of p38 MAP kinase inhibitors with high anti-inflammatory activity have been reported ${ }^{19}$. O-substituted benzophenones could provide more complex polyaromatics, for example, pentamethoxy-diphenylmethanone ${ }^{20}$, and similarly substituted benzophenones could lead to useful synthons for fullerenes. Generally, they are prepared via Friedel-Crafts acylation of aromatic compounds ${ }^{21-23}$, however this paper describes the Grignard reaction for their synthesis, (Scheme 1)
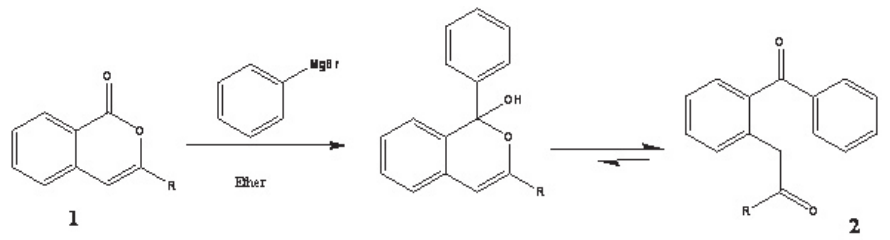

Scheme 1: Synthesis of o-substituted benzophenones from isocoumarins

\section{EXPERIMENTAL}

\section{Materials and Methods}

Chemicals were purchased from Aldrich Chemical Co. and used as such without further purification. TLC was performed on silica plates with visualization by UV-light. Melting points were taken in open capillary tubes and corrected with reference to benzoic acid. IR spectra in $\mathrm{KBr}$ pellets were recorded on Nucon Infrared spectrophotometer. ${ }^{1} \mathrm{H}$ NMR $(400 \mathrm{MHz})$ and ${ }^{13} \mathrm{C}$ NMR (100 MHz) spectra were recorded on a Bruker $400 \mathrm{MHz}$ spectrometer in $\mathrm{CDCl}_{3}$ or DMSO (with TMS for ${ }^{1} \mathrm{H}$ NMR and DMSO for ${ }^{13} \mathrm{C}$ NMR as internal references). LC-MS analyses were performed with LCMS-Agilent- 1100 series Ion Trap. Mass spectra were recorded on ABI QSTAR XL ESI-TOF Mass spectrometer (Sciex Model) operating at $70 \mathrm{eV}$ using fast atom bombardment technique. Elemental analyses of all compounds were performed on Elementar Vario Micro CHNS analyzer.

Synthesis of o-substituted benzophenones from isocoumarins (1a-f)

Synthesis of the hitherto title compounds is as outlined in Scheme 1. Grignard reaction of 3-substituted isocoumarins (1) in anhydrous ether afforded substituted benzophenones (2) in modest to good yield. The results have been tabulated as Table 1 . All products were characterized by IR, ${ }^{1} \mathrm{H}$ NMR and mass spectrophotometers.

Typical Procedure:

To a stirred solution of 3- $p$ Methoxyphenylisocoumarin, 1a $(20 \mathrm{mmol})$ in anhydrous ether $(50 \mathrm{ml})$ maintained at $-78^{\circ} \mathrm{C}$ in dry ice-solvent bath, phenyl magnesium bromide in ether $(40 \mathrm{mmol})$ was added. Magnetic stirring was continued for $4 \mathrm{hrs}$ and completion of the reaction was monitored by TLC using hexane and ethyl acetate 95:5 as eluant. The reaction mixture was worked up at room temperature by treating with aq. $\mathrm{NH}_{4} \mathrm{Cl}$ and then extracted with ethyl acetate. The crude product was purified by silica gel column (using hexane and ethyl acetate) as eluant. The product, 2a was obtained with $77 \%$ yield. The product obtained was characterized by IR, LCMS, HRMS, ${ }^{1} \mathrm{H}-\mathrm{NMR}$ and ${ }^{13} \mathrm{C}$-NMR techniques. The reaction of Phenyl magnesium bromide with various isocoumarins, 1a-f was tabulated (Table 1).

Table 1: Synthesis of o-substituted benzophenones from 3-substituted isocoumarins and phenyl magnesium bromide.

\begin{tabular}{|l|c|}
\hline \multicolumn{1}{|c|}{ Isocoumarins ${ }^{\mathrm{a}}, \mathbf{( 1 )} /$ Products (2) } & Yield $^{\mathbf{b}} \mathbf{0}$ \\
\hline$p-\mathrm{OCH}_{3}-\mathrm{C}_{6} \mathrm{H}_{4},{ }^{\mathrm{a}}$ & 77 \\
\hline$p-\mathrm{CH}_{3}-\mathrm{C}_{6} \mathrm{H}_{4}, \mathrm{~b}$ & 81 \\
\hline$p-\mathrm{NO}_{2}-\mathrm{C}_{6} \mathrm{H}_{4}, \mathrm{c}$ & 73 \\
\hline$p-\mathrm{Cl}_{-} \mathrm{C}_{6} \mathrm{H}_{4}, \mathrm{~d}$ & 72 \\
\hline $\mathrm{CH}_{3} \mathrm{CH}_{2} \mathrm{CH}_{2} \mathrm{CH}, \mathrm{e}$ & 80 \\
\hline $1-\mathrm{Cyclohexenyl,}$ & 82 \\
\hline
\end{tabular}

asocoumarin, 1a-f (20mmol) in anhydrous ether $(50 \mathrm{ml})$, phenylmagnesium bromide, $(40 \mathrm{mmol})$, stirred at $-78^{\circ} \mathrm{C}$ in dry ice-solvent bath for 4 hours, bisolated yield, 2.

The spectral data of compounds, $\mathbf{2 a - 2} \mathbf{f}$ is given below.

2-(2-Benzoylphenyl)-1-(4-methoxy phenyl) ethanone (2a) Gummy solid, IR cm ${ }^{-1} 3053.6,2953.6,2984.6,2867.8,1724.8,1676.4 ; \mathrm{H}^{1} \mathrm{NMR}(400 \mathrm{MHz}$, $\left.\mathrm{CDCl}_{3}\right) 3.79\left(\mathrm{~s}, 3 \mathrm{H}, \mathrm{OCH}_{3}\right), 4.55\left(\mathrm{~s}, 2 \mathrm{H}, \mathrm{CH}_{2}\right) 6.86-6.89(\mathrm{~m}, 2 \mathrm{H}), 7.33-7.55(\mathrm{~m}$, $7 \mathrm{H}), 7.81-7.83(\mathrm{~m}, 2 \mathrm{H}), 7.91-9.36(\mathrm{~m}, 2 \mathrm{H}) ; \mathrm{C}^{13} \mathrm{NMR}\left(100 \mathrm{MHz}, \mathrm{CDCl}_{3}\right) 39.43$ $(\mathrm{OCH}), 42.87(\mathrm{COCH}), 126.52,128.33,128.76,129.73,130.58,130.64$, $131.59,132.63,132.74,134.48,137.86,138.25,139.39$ (13C, Aryl), 196.18 $(\mathrm{C}=\mathrm{O}) 198.57\left(\mathrm{COCH}_{2}\right)$; LCMS: m/e 331.31; HRMS [M $\left.{ }^{+}\right]$330. 2921, Mol. Formula $\mathrm{C}_{22} \mathrm{H}_{18} \mathrm{O}_{3}$, Mol. Wt.: 330.38, Calculated C, 79.98; H, 5.49; O, 14.53 found $\mathrm{C}, 79.79 ; \mathrm{H}, 5.57 ; \mathrm{O}, 14.63$.

2-(2-Benzoylphenyl)-1-(4-methyl phenyl) ethanone (2b) Gummy solid, IR cm ${ }^{-1} 3068.6,2961.5,2979.8,2872.8,1718.5,1669.4 ; \mathrm{H}^{1} \mathrm{NMR}(400 \mathrm{MHz}$, $\left.\mathrm{CDCl}_{3}\right) 2.38\left(\mathrm{~s}, 3 \mathrm{H}, \mathrm{CH}_{3}\right), 4.59\left(\mathrm{~s}, 2 \mathrm{H}, \mathrm{CH}_{2}\right) 7.20-7.22(\mathrm{~d}, \mathrm{~J}=8 \mathrm{~Hz}, 2 \mathrm{H}), 7.32$ $7.36(\mathrm{~m}, 2 \mathrm{H}), 7.42-7.46(\mathrm{t}, \mathrm{J}=8.0 \mathrm{~Hz}, 3 \mathrm{H}), 7.47-7.51(\mathrm{~d}, \mathrm{~J}=7.6 \mathrm{~Hz}, 1 \mathrm{H})$, 7.54- $7.58(\mathrm{t}, \mathrm{J}=7.6 \mathrm{~Hz}, 1 \mathrm{H}), 7.81-7.86(\mathrm{~d}, \mathrm{~J}=8.4 \mathrm{~Hz}, 4 \mathrm{H}) ; \mathrm{C}^{13} \mathrm{NMR}(100$ $\left.\mathrm{MHz}, \mathrm{CDCl}_{3}\right) 21.60\left(\mathrm{CH}_{3}\right), 42.95\left(\mathrm{COCH}_{2}\right), 126.18,128.22,128.38,129.19$, 
$130.15,130.40,130.86,132.00,132.76,134.33,134.83,137.92,138.40143 .79$ (14C, Aryl), 196.71, (C=O) $198.34\left(\mathrm{COCH}_{2}\right)$; LCMS: m/e 315.3; HRMS [M $\left.{ }^{+}\right]$ 314.5187, Mol. Formula $\mathrm{C}_{22} \mathrm{H}_{18} \mathrm{O}_{2}$, Mol. Wt.: 314.38 .

2-(2-Benzoylphenyl)-1-(4-nitro phenyl) ethanone (2c) Gummy solid, IR cm ${ }^{-1} 3046.3,2987.8,2964.7,2867.4,1727.4,1678.4 ; \mathrm{H}^{1} \mathrm{NMR}$ (400 MHz $\left.\mathrm{CDCl}_{3}\right) 4.61\left(\mathrm{~s}, 2 \mathrm{H}, \mathrm{CH}_{2}\right)$ 7.38-7.42 (m, 4H), 7.45-7.49 (m, 3H), 7.50-7.51 (d, $\mathrm{J}=7.61 \mathrm{~Hz}, 1 \mathrm{H}), 7.53-7.62(\mathrm{t}, \mathrm{J}=7.7 \mathrm{~Hz}, 1 \mathrm{H}), 7.81-7.83(\mathrm{~d}, \mathrm{~J}=9.59 \mathrm{~Hz}, 2 \mathrm{H})$ 7.87- $7.89(\mathrm{~d}, \mathrm{~J}=10.7 \mathrm{~Hz}, 2 \mathrm{H})$; $\mathrm{C}^{13} \mathrm{NMR}\left(50 \mathrm{MHz}^{\mathrm{CDCl}}\right) 43.17\left(\mathrm{COCH}_{2}\right)$, $126.41,128.18,128.77,129.62,130.52,130.58,131.28,132.46,132.84$, 134.32, 137.83, 138.29, 139.57 (13C, Aryl), 196.26, (C=O) $199.37\left(\mathrm{COCH}_{2}\right)$; LCMS: m/e 346.23; HRMS $\left[\mathrm{M}^{+}\right]$346. 0129. Mol. Formula $\mathrm{C}_{21} \mathrm{H}_{15} \mathrm{NO}_{4}, \mathrm{Mol}$. Wt.: 345.35

2-(2-Benzoylphenyl)-1-(4-chloro phenyl) ethanone (2d) Gummy solid, IR $\mathrm{cm}^{-1} 3058.8,2967.5,2983.8,2863.4,1728.5,1659.3,837,641 ; \mathrm{H}^{1} \mathrm{NMR}$ $\left(400 \mathrm{MHz}, \mathrm{CDCl}_{3}\right) 4.57\left(\mathrm{~s}, 2 \mathrm{H}, \mathrm{CH}_{2}\right)$ 7.34-7.41 (m, 4H), 7.43-7.47 (m, 3H) $7.48-7.49(\mathrm{~d}, \mathrm{~J}=7.6 \mathrm{~Hz}, 1 \mathrm{H}), 7.50-7.59$ (t, J=7.6 Hz, 1H), 7.79- 7.81 (d, J= $9.6 \mathrm{~Hz}, 2 \mathrm{H}), 7.88-7.90(\mathrm{~d}, \mathrm{~J}=10.8 \mathrm{~Hz}, 2 \mathrm{H}) ; \mathrm{C}^{13} \mathrm{NMR}\left(50 \mathrm{MHz}, \mathrm{CDCl}_{3}\right) 43.13$ $\left(\mathrm{COCH}_{2}\right), 126.40,128.26,128.84,129.69,130.35,130.41,131.05,132.05$, $132.83,134.45,137.87,138.07,139.46$ (13C, Aryl), 195.96, (C=O) 198.29 $\left(\mathrm{COCH}_{2}\right)$; Mol. Formula $\mathrm{C}_{21} \mathrm{H}_{15} \mathrm{ClO}_{2}$ Mol. Wt.: 334.80, LCMS: 335.39 HRMS $\left[\mathrm{M}^{+}\right]$334.5289, Mol. Formula $\mathrm{C}_{21} \mathrm{H}_{15} \mathrm{ClO}_{2}$, Mol. Wt.: 334.80

1-(2-benzoylphenyl)hexan-2-one, (2e) Gummy solid, IR cm-13062.7, 2956.7, 2991.0, 2871.8, 1716.5, 1660.6; $\mathrm{H}^{1} \mathrm{NMR}\left(400 \mathrm{MHz}, \mathrm{CDCl}_{3}\right) 0.78$ $0.79\left(\mathrm{t}, 3 \mathrm{H}, \mathrm{CH}_{3}\right), 1.2\left(\mathrm{~m}, 2 \mathrm{H}, \mathrm{CH}_{2}\right), 1.41\left(\mathrm{~m}, 2 \mathrm{H}, \mathrm{CH}_{2}\right), 2.36-2.39(\mathrm{t}, 2 \mathrm{H}$ $\left.\mathrm{CH}_{2}\right), 3.88\left(\mathrm{~s}, 2 \mathrm{H}, \mathrm{CH}_{2}\right), 7.22(\mathrm{~m}, 2 \mathrm{H}), 7.31-7.34(\mathrm{~m}, 1 \mathrm{H}), 7.36-7.42(\mathrm{~m}, 3 \mathrm{H})$, 7.48-7.52 (m, 1H), 7.71-7.73 (m, 2H); $\mathrm{C}^{13} \mathrm{NMR}\left(50 \mathrm{MHz}, \mathrm{CDCl}_{3}\right) 13.8\left(\mathrm{CH}_{3}\right)$, $22.3\left(\mathrm{CH}_{3} \mathrm{CH}_{2}\right), 25.8\left(-\mathrm{CH}_{2} \mathrm{CH}_{2}\right), 42.8\left(\mathrm{CH}_{2} \mathrm{CO}\right), 47.2\left(\mathrm{COCH}_{2}, 126.1,128.2\right.$ $130.0,130.8,131.9,132.7,134.4,137.8,137.9$ (9C, Aryl), 198.0, (C=O) 207.5 $\left(\mathrm{COCH}_{2}\right)$; LCMS: 281.43; HRMS $\left[\mathrm{M}^{+}\right]$280.3589. Mol. Formula $\mathrm{C}_{19} \mathrm{H}_{20} \mathrm{O}_{2}$, Mol. Wt.: 280.36, Calculated C, 81.40; H, 7.19; O, 11.41, found C, 81.53; H, 7.32; O, 11.53.

2-(2-benzoylphenyl)-1-cyclohex-1-en-1-ylethanone(2f) Gummy solid, IR cm ${ }^{-1} 3062.8,2955.3,2989.4,2867.8,1724.8,1662.3 ; \mathrm{H}^{1} \mathrm{NMR}(400 \mathrm{MHz}$ $\left.\mathrm{CDCl}_{3}\right)$ 1.54-1.58 (5H, m) 2.14-2.18 ( m, 4H), $4.25\left(\mathrm{~s}, 2 \mathrm{H}, \mathrm{CH}_{2}\right), 6.91-6.93(\mathrm{~s}$, $1 \mathrm{H}), 7.31-7.38(\mathrm{~m}, 2 \mathrm{H}) 7.42-,7.47(\mathrm{~m}, 3 \mathrm{H}), 7.54-7.56(\mathrm{~m}, 1 \mathrm{H}), 7.79-7.87(\mathrm{~m}$, $2 \mathrm{H}) ; \mathrm{C}^{13} \mathrm{NMR}\left(50 \mathrm{MHz}, \mathrm{CDCl}_{3}\right)$ 21.43, 21.85, 23.18, 26.04, $41.42\left(\mathrm{COCH}_{2}\right)$ $115.23,125.87,129.30$ (3C, Alicyclic), 129.74, 130.25, 130.61, 131.83, 132.68, 135.08, 137.80, 138.29, 138.70, 140.35 (10C, Aryl), $197.86(\mathrm{C}=\mathrm{O}), 198.43$ $\left(\mathrm{COCH}_{2}\right)$; HRMS [M+] 304.5713. Mol. Formula $\mathrm{C}_{21} \mathrm{H}_{20} \mathrm{O}_{2}$, Mol. Wt.: 304.38, Calculated C, 82.86; H, 6.62; O, 10.51, found C, 82.74; H, 6.58; O, 10.43.

\section{RESULTS AND DISCUSSION}

The 3-substituted isocoumarins required for our reaction were prepared by reported procedure ${ }^{10-12}$. Purified isocoumarins was then allowed to react with Phenyl magnesium bromide in presence of anhydrous ether at $-78^{\circ}$ $\mathrm{C}$ to afford the corresponding hemiacetal which undergo isomerism to give the corresponding diketone derivatives, (Scheme 1; Table 1). The reaction producing o-substituted benzophenone derivatives by a simple and an efficien route gave modest to good yields. Products of the reaction have been isolated, purified and characterized by various spectral techniques such as IR, LC-MS, ${ }^{1} \mathrm{H}-\mathrm{NMR}$ and ${ }^{13} \mathrm{C}$-NMR techniques. It is noteworthy that among various 3substituted isocoumarins (see Table 1), those with electron rich groups accelerated the Grignard reaction and produced higher yield compared to that with electron deficient groups

\section{Mass spectra and elemental analysis}

The high resolution mass spectra of diketones showed a molecular ion peak $\mathrm{M}^{+}$. The molecular ion peak for $\mathbf{2 a}$ was observed at $\mathrm{m} / \mathrm{z}=330.2921$. This is also supported by the mass spectra of the other compounds, 2b-2f. Elemental analysis values are in close agreement with the values calculated from molecular formula assigned to these compounds, which is further supported by the LCMS studies of representative compounds.

\section{IR and NMR spectra}

In IR spectrum, products gave peaks at around $1700-1750 \mathrm{~cm}^{-1}$ and in ${ }^{13} \mathrm{C}$ NMR there are two peaks at around 190-200ppm indicating the presence of two ketogroups which in turn confirming isomerism of hemiacetal, (Grignard addition product of isocoumarins and Phenylmagnesium bromide) to the final ketoproduct.

\section{CONCLUSIONS}

The C-C bond formation was successfully carried out by Grignard reaction of isocoumarins giving rise to the diketoderivatives. These diketo derivatives may be used as synthetic intermediates in Aldol reaction (our laboratory group's research area) to give biologically important polyaromatic hydrocarbons and in other reaction, may pave their way in synthesis of useful products

\section{ACKNOWLEDGEMENT}

The authors wish to express their gratitude to SAIF, Indian Institute of Science, Bangalore; Spic Science foundation, Tuticorin and Syngene International Limited, Bangalore for their support of NMR, HRMS and elemental analysis facilities.

\section{REFERENCES}

1. H. Matsuda, H. Shimoda, M. Yoshikawa, Bioorg. Med. Chem. 7, 1445 (1999)

2. H. Shimoda, H. Matsuda, J. Yamahara, M. Yoshikawa, Biol. Pharm. Bull. 21, 809 (1998)

3. A. C. Whyte, J. B. Gloer, J. A. Scott, D. J. Mallock, Nat. Prod. 59, 765 (1996)

4. T. Furuta, Y. Fukuyama, Y. Asakawa, Phytochemistry 25, 517 (1986)

5. R. D. Barry, Chem. Rev. 64, 229 (1964)

6. R. S. Mali, K. N. Babu, J. Org. Chem. 63, 2488 (1998) and references cited therein.

7. Nitin T. Patil, F. Nawaz Khan, Y. Yamamoto, Tetrahedron Lett. 45, 8497 (2004)

8. F. Nawaz Khan, R. Jayakumar, C.N. Pillai, J.Mol.Cat. A, Chemical 195, 139 (2003)

9. F. Nawaz Khan, R. Jayakumar, C.N. Pillai, Tetrahedron Lett. 43, 6807 (2002)

10. Venkatesha R. Hathwar, P. Manivel, F. Nawaz Khan, T.N. Guru Row, Acta Cryst. E63, o3707 (2007)

11. Venkatesha R. Hathwar, P. Manivel, F. Nawaz Khan, T.N. Guru Row, Acta Cryst. E63, o3708 (2007)

12. S. Syed Tajudeen, F. Nawaz Khan, Synth. Commun. 37, 3649 (2007)

13. G.A. Olah. Friedel-Crafts and Related Reaction, Vol. III, Interscience, New York, 1964, Part I.

14. H. Szmant. Organic Building Blocks of the Chemical Industry, Wiley, New York, 1989.

15. J. March. Advanced Organic Chemistry, fourth ed., Wiley, New York, 1992.

16. J. Jiri, P. Miroslav, P. Josef, W. Stanislav. Csech CS. 271,185 (1991), (Chem. Abstr. 1992, 117, 170994d)

17. M. Williams, E.A. Kowaluk and S.P. Arneric, J. Med. Chem. 42, 1481 (1999)

18. I. Yoshiyuki, K. Miwako, K. Shusuke, Jpn. Kokai Tokkya Koho JP. 1991, 03,209,318, ( Chem. Abstr. 1992, 116, 99311a).

19. E.R. Ottosen, M.D. Sorensen, F. Bjorkling, T. Skak-Nielsen, M.S. Fjording, H. Aaes and L. Binderup, J. Med. Chem. 46, 5651 (2003)

20. D. Walker, J.D. Hiebert, ChemRev. 67, 153 (1967)

21. F. Karrer, H. Meier, A. Pascual, J. Fluorine Chem. 103, 81 (2000)

22. C.G. Jia, M.Y. Huang, Y.Y. Jiang, Chin. J. Chem. 5, 452 (1993)

23. K. Kowalski, J. Zakrzewski, L. Jerzykiewicz, J. Organomet. Chem. 690, 1474 (2005). 